TRANSACTIONS OF THE

AMERICAN MATHEMATICAL SOCIETY

Volume 359, Number 5, May 2007, Pages 2021-2041

S 0002-9947(06)04315-7

Article electronically published on December 15, 2006

\title{
A UNIQUE GRAPH OF MINIMAL ELASTIC ENERGY
}

\author{
ANDERS LINNÉR AND JOSEPH W. JEROME
}

\begin{abstract}
Nonlinear functionals that appear as a product of two integrals are considered in the context of elastic curves of variable length. A technique is introduced that exploits the fact that one of the integrals has an integrand independent of the derivative of the unknown. Both the linear and the nonlinear cases are illustrated. By lengthening parameterized curves it is possible to reduce the elastic energy to zero. It is shown here that for graphs this is not the case. Specifically, there is a unique graph of minimal elastic energy among all graphs that have turned 90 degrees after traversing one unit.
\end{abstract}

\section{INTRODUCTION}

1.1. Elastic energy. Let $\kappa$ denote the signed curvature of a sufficiently smooth regular curve $\gamma:[0, L] \rightarrow \mathbb{R}^{2}$, and assume $\gamma$ is parameterized by arc-length. The length of $\gamma$ is $L$, and its elastic energy is proportional to

$$
E(\gamma)=\int_{0}^{L} \kappa^{2}(s) d s .
$$

Daniel Bernoulli suggested the quantity (1.1) in a letter to Euler, dated 1742; see page 507 in 5. The Willmore functional is a two-dimensional analogue of (1.1) where the mean curvature of a surface takes the place of $\kappa$. This explains the phrase 'one-dimensional Willmore equation' sometimes used for the Euler-Lagrange equation satisfied by $\kappa$; see [4 for a recent example.

It is clear that each straight line segment is a global minimum of $E$. When boundary conditions are imposed that preclude straight segments, the existence of a minimum is no longer guaranteed. A simple example of this occurs when the length $L$ is free to vary and $\gamma(L)=\gamma(0)$ is required. Parameterized circles of radius $r$ satisfy this periodic boundary condition. Moreover, their elastic energy $E$ is $2 \pi / r$, which tends to 0 as the radius increases without bound. A different way to preclude the straight line is analyzed in [2, where it is shown that $E(\gamma)$ has its greatest lower bound equal to zero when restricted to parameterized curves with endpoints $\gamma(0)=p_{0}, \gamma(L)=p_{2}$ prescribed together with at least one more interpolation point $\gamma\left(s_{1}\right)=p_{1}$. Again it is important that the length $L$ is arbitrary, and the case of

Received by the editors January 12, 2005

2000 Mathematics Subject Classification. Primary 58E25, 49J30; Secondary 58Z05.

Key words and phrases. Elastic energy, variable length, pendulum equation, Pontrjagin, maximum principle, phase constraint, optimal graph, one-dimensional Willmore equation.

The research for this paper was essentially completed during the first author's sabbatical year at Northwestern.

The second author's research was supported in part by NSF grant DMS-0311263.

(C)2006 American Mathematical Society Reverts to public domain 28 years from publication 
interest is when the three prescribed points are not all on the same straight line. Yet another possibility is discussed in [9], where it is shown that the greatest lower bound is zero when both the tangent directions $\gamma^{\prime}(0)=v_{0}, \gamma^{\prime}(L)=v_{1}$ and the endpoints $\gamma(0)=p_{0}, \gamma(L)=p_{1}$ are prescribed. This time the interesting cases occur when at least one of the vectors $p_{1}-p_{0}, v_{0}, v_{1}$ points in a direction different from the rest. All these examples share the trait that the minimizing sequences ultimately consist of curves that are not graphs. This suggests that among graphs of arbitrary lengths there is a global minimum. The purpose of this article is to analyze this issue when the curves satisfy the simplest boundary conditions that preclude the straight line segment.

1.2. Critical points. In the exceptional case when the derivative $D E(\gamma) v$ vanishes in all admissible directions $v, \gamma$ is considered a critical point of $E$. A global minimum is always a critical point, but not all critical points correspond to a global minimum. An example of this is seen, perhaps surprisingly, in the case of unconstrained length together with the boundary conditions $\gamma(0)=0, \gamma(L)=p \neq 0$. The straight line between the two endpoints is a global minimum of $E$, but there is also a countable collection of distinct critical points $\gamma_{i}$ with $E\left(\gamma_{i}\right) \rightarrow+\infty$, [10. Interestingly, in this case each critical $\gamma_{i}$ has the same length.

As mentioned already, there is no global minimum in the case of periodic boundary conditions and unconstrained length, and more generally, there are no critical points; see [10. When the length $L$ is bounded (or fixed), there is always a global minimum for $E$. For instance, in [7] it is shown that, when there is any number of prescribed interpolation points, there always exists a minimizing $\gamma$.

An alternative way to guarantee existence is to add a penalty term $\nu L$ to $E(\gamma)$, with $\nu>0$. This time there is a global minimum in the space of, say periodic, $\gamma:[0, L] \rightarrow M$, where $M$ is any Riemannian manifold 8 . Incidentally, the negative gradient trajectories of $E$ do not in general stay inside the collection of graphs when $M$ is the Euclidean plane. Another curiosity is that, in arbitrary two-dimensional $M$ and with $\nu=0$, the quantity $L(E+2 L)$ is invariant along the negative gradient trajectories of $E$, provided no constraints are imposed on any of the tangent directions; see [11].

The main result of the present article is to show that, when the length $L$ is variable and the curves are required to be graphs, there are boundary conditions that preclude line segments where $E$, nevertheless, has a global minimum.

1.3. Organization. The article presents several results of independent interest and with different implications for the main result. The domain of curves considered is chosen to keep the presentation as explicit as possible. With this in mind, the left endpoint is fixed with horizontal tangent direction. The right endpoint is required to be on a vertical line and have a vertical tangent direction there. The length is variable, and the tangents are not allowed to go beyond vertical upwards or downwards. By using this domain, a general inequality is proved that implies that the greatest lower bound of $E(\gamma)$ is greater than zero; see Section 2 . The setting for this is a 'linear' version of the more complicated problems that follow. For instance, the latter problems have to contend with a nonlinear isoperimetric constraint. This causes a convex domain to be replaced by a nonflat infinite-dimensional manifold with boundaries, and potential solutions are given by a nonlinear differential equation as opposed to a linear one. 
The next result shows that there is a countably infinite family of critical graphs; see Section 3. This holds despite the fact that there is no assumption regarding the existence of a minimum, nor are the curves a priori assumed to be graphs.

In Section 4 it is proved that when the length is fixed, there is always a minimal curve, even when the tangent directions are restricted to never go beyond vertical. The strategy is to consider each fixed length separately and gather enough information to draw the appropriate conclusion when there is no constraint on the length. It turns out that it is not necessary to have this kind of comprehensive knowledge to establish the main result. Such a detailed description does, however, serve other purposes. In particular, the proof of this result and its preceding linear counterpart point toward a general methodology for dealing with nonlinear functionals that are formed as products of two integrals rather than the usual single integral, or sums of such, as is typical in the calculus of variations.

Finally, the main result is proved in Section 4, There is a unique explicit solution of minimal elastic energy among graphs with no restriction on the length. To remove the trivial case of $y \equiv 0$, it is assumed that the tangent line is horizontal at the left endpoint and vertical at the right endpoint. All the data regarding this exceptional curve are given in terms of elliptic functions. It is then seen that the minimal elastic energy is more than three times as large as the lower bound given by the analysis of the linear analogue.

We thank the referee for the very thoughtful, thorough and helpful report.

\section{Positive greatest Lower Bound}

Recall that, when the length is variable, the greatest lower bound of the elastic energy is zero, even when the straight segment is precluded by the boundary conditions or points of interpolation. Our first theorem establishes an inequality that, for one particular set of boundary conditions, implies a strictly positive greatest lower bound when the curves are required to be graphs. Specifically, let $W_{1}^{2}[0,1]$ denote the Sobolev space of real-valued absolutely continuous functions on $[0,1]$ with derivative in $L^{2}[0,1]$. The inner product in $W_{1}^{2}[0,1]$, denoted by $\langle$,$\rangle , is chosen so$ that the norm satisfies $\|x\|^{2}=x^{2}(0)+\int_{0}^{1} \dot{x}^{2}(t) d t$.

Theorem 2.1. Consider all functions $x \in W_{1}^{2}[0,1]$ subject to $x(0)=1, x(1)=0$, and $x(t) \geqslant 0$. The following inequality always holds:

$$
\int_{0}^{1} x(t) d t \int_{0}^{1} \dot{x}^{2}(t) d t \geqslant 4 / 9
$$

with equality if and only if for some $a$, such that $0<a \leqslant 1$,

$$
x(t)=x_{a}(t):= \begin{cases}q(t), & 0 \leqslant t \leqslant a, \\ 0, & a<t \leqslant 1,\end{cases}
$$

where $q(t)=(a-t)^{2} / a^{2}$ is a quadratic polynomial in $t$ such that $q(0)=1, q(a)=0$, and $\dot{q}(a)=0$.

Proof. A quick calculation shows that $\int_{0}^{1} x_{a}(t) d t=\frac{a}{3}$, and $\int_{0}^{1} \dot{x}_{a}^{2}(t) d t=\frac{4}{3 a}$. Let $c$ be a fixed positive number and consider only functions that satisfy $G(x):=$ $\int_{0}^{1} x(t) d t=c$. The linear $G$ together with the other constraints determine a closed convex domain. For each $c$, the task is to minimize $\int_{0}^{1} \dot{x}^{2}(t) d t=\|x\|^{2}-1$, which is equivalent to a minimum norm problem. It follows that there exists, in fact, a 
unique minimum. Observe that the derivative of $G$ in the direction $v$ is given by $D G(x) v=\int_{0}^{1} v(t) d t$. Integration by parts shows that the gradient $\nabla G$ is given by $\nabla G(x)=1+t-t^{2} / 2$, which is verified by the equality $\langle\nabla G(x), v\rangle=D G(x) v$. Let $F(x)=\frac{1}{2} \int_{0}^{1} \dot{x}^{2}(t) d t$ and observe that $D F(x) v=\int_{0}^{1} \dot{x}(t) \dot{v}(t) d t$. It is immediate that the gradient is given by $\nabla F(x)=x(t)-x(0)$, which satisfies $\langle\nabla F(x), v\rangle=D F(x) v$ as required. If the constraint $x(t) \geqslant 0$ is ignored, then each critical point must satisfy

$$
x(t)-1-\lambda\left(1+t-t^{2} / 2\right)-\mu_{0}-\mu_{1}(1+t)=0,
$$

for some real multipliers $\lambda, \mu_{0}$ and $\mu_{1}$.

The right and left endpoint constraints, together with the isoperimetric constraint, lead to a linear system that must be satisfied by the three multipliers. The matrix form of this system is

$$
\left[\begin{array}{lll}
1 & 1 & 1 \\
3 / 2 & 1 & 2 \\
4 / 3 & 1 & 3 / 2
\end{array}\right]\left[\begin{array}{l}
\lambda \\
\mu_{0} \\
\mu_{1}
\end{array}\right]=\left[\begin{array}{l}
0 \\
-1 \\
c-1
\end{array}\right],
$$

and its solution is $\lambda=-6+12 c, \mu_{0}=4-6 c, \mu_{1}=2-6 c$.

The unique critical point is $x(t)=(1-t)(1+(6 c-3) t)$. When $c=1 / 2$, this function corresponds to a straight line that is never negative on the domain $[0,1]$. In all other cases, this quadratic function is zero when $t=1$ and $1 / t=3(1-2 c)$. When $c>1 / 2$, the curve is a downward parabola that satisfies $x(t) \geqslant 0$ in $[0,1]$. When $1 / 3 \leqslant c<1 / 2$, the curve is an upward parabola that satisfies $x(t) \geqslant 0$ in $[0,1]$. When $0<c<1 / 3$, the curve violates $x(t) \geqslant 0$ in $[0,1]$. In all cases but the last, this reasoning produces the minimum because $x$ 'by coincidence' satisfies $x(t) \geqslant 0$. Before dealing with the case $0<c<1 / 3$, note that

$$
\int_{0}^{1} x(t) d t \int_{0}^{1} \dot{x}^{2}(t) d t=4 c\left(1-3 c+3 c^{2}\right) .
$$

The derivative with respect to $c$ is given by $4(1-3 c)^{2}$, which shows that the minimum for cases considered thus far is when $c=1 / 3$.

The case $0<c<1 / 3$ is covered by the Pontrjagin maximum principle for phase constraints as analyzed in [6], and the same notation is used here. The phase space is augmented by one dimension in order to accommodate the isoperimetric constraint $G(x)=c$. Let $x_{1}=x$ and $x_{2}=\int_{0}^{t} x$. This produces the endpoint constraints $x_{1}(0)=1, x_{1}(1)=0$, and $x_{2}(0)=0, x_{2}(1)=c$. It suffices to introduce a one-dimensional unrestricted control $u$ subject to the system

$$
\begin{aligned}
& \dot{x}_{1}=\dot{x}=u, \\
& \dot{x}_{2}=x=x_{1} .
\end{aligned}
$$

To properly apply Theorem 1, p. 234 of [6] of all the information is collected into functions. The objective has the integrand $f\left(t, x_{1}, x_{2}, u\right)=u^{2} / 2$. The governing system is recorded in the two-dimensional $\varphi\left(t, x_{1}, x_{2}, u\right)=\left(u, x_{1}\right)$. The affine endpoint constraints are in $h_{0}\left(x_{1}(0), x_{2}(0)\right)=\left(x_{1}(0)-1, x_{2}(0)\right)$, and $h_{1}\left(x_{1}(1), x_{2}(1)\right)=$ $\left(x_{1}(1), x_{2}(1)-c\right)$. Finally, the phase constraint is in $g\left(t, x_{1}(t), x_{2}(t)\right)=-x_{1}(t) \leqslant 0$. The Pontrjagin function is given by

$$
P\left(t, x_{1}, x_{2}, u, p_{1}, p_{2}, \lambda_{0}\right)=p_{1} u+p_{2} x_{1}-\lambda_{0} u^{2} / 2 .
$$


The Hamiltonian is given by

$$
H\left(t, x_{1}, x_{2}, p_{1}, p_{2}, \lambda_{0}\right)=\frac{p_{1}^{2}}{2 \lambda_{0}}+p_{2} x_{1} .
$$

The adjoint vector equation is the integral equation

$$
\left(p_{1}(t), p_{2}(t)\right)=-\left(l_{1}^{1}, l_{1}^{2}\right)+\left(\int_{t}^{1} p_{2}(\tau) d \tau, 0\right)+\left(\int_{t}^{1} d \mu, 0\right),
$$

with $\left(p_{1}(0), p_{2}(0)\right)=\left(l_{0}^{1}, l_{0}^{2}\right)$. Here $l_{i}^{j}$ are real numbers, $\tau$ a standard parameter of integration, and $\mu$ a nonnegative regular measure supported on the set $T=$ $\{t: x(t)=0\}$. The Pontrjagin function and the Hamiltonian should be equal for almost all $t \in[0,1]$, so $u=p_{1} / \lambda_{0}$ for almost all $t$. In general, $p_{i}$ is only assumed to be of bounded variation and continuous from the left. Here the adjoint equation implies that $p_{2}$ is constant, and hence $\lambda_{0} u=p_{1}(t)=-l_{1}^{1}+p_{2}(1-t)+\int_{t}^{1} d \mu$. Since $\dot{x}=u$, one integration shows that $x$ is quadratic as long as $t \notin T$. The possibility $\lambda_{0}=0$ forces $p_{2}=0$, and the measure $\mu$ must be supported at the single point $t=1$. With $l_{1}^{1}=\mu_{1}[\{1\}] \neq 0$ it is seen that not all multipliers are zero. This is due to the fact that the constraints $x(t) \geq 0$ and $x(1)=0$ are redundant at $t=1$. When $0<c<1 / 3$ it is seen above that there must be some $t^{\prime}<1$ such that $x\left(t^{\prime}\right)=0$, so $T$ is not a single point.

The derivative of an optimal function must be bounded. This can be shown using variational techniques; see www.math.niu.edu/ alinner, which also covers the nonlinear case of Theorem 4.2.

To proceed, first examine all curves of the form one quadratic piece followed by a single straight piece with $x(t)=0$. For a fixed $c$, and a quadratic on the interval $[0, a]$, the conditions $x(0)=1, x(a)=0, \int_{0}^{a} x(t) d t=c$, and $x(t) \geqslant 0$, produce a family of curves $x(t)=(a-t)\left(a^{2}-3 a t+6 c t\right) / a^{3}$ that depend on $a$ and $c$ where $a \leqslant 3 c$ is required to ensure $x(t) \geqslant 0$. The derivative of $G(x) F(x)$ with respect to $a$ is $-2 c(a-3 c)^{2} / a^{4}$. It follows that for each $c$ the optimal $a$ is the largest admissible $a=3 c$. The quadratic piece has the form $Q(t)=\frac{(t-3 c)^{2}}{9 c^{2}}$. Now it turns out that $\int_{0}^{3 c} Q(t) d t \int_{0}^{3 c} \dot{Q}^{2}(t) d t=4 / 9$. To finish the argument, imagine a nonnegative curve with more than one quadratic piece. Since $x(0)=1$, the initial piece is quadratic with $x(t)>0$ on say the maximal interval $\left[0, t^{\prime}\right)$. Replace the imagined curve by a curve with the same initial quadratic piece and zero on $\left[t^{\prime}, 1\right]$. Because $x(t) \geqslant 0$, the quantity $\int_{0}^{1} x(t) d t \int_{0}^{1} \dot{x}^{2}(t) d t$ is smaller for the new curve, but it is certainly not less than $4 / 9$.

Remark. Suppose the lower half-plane is regarded as an obstacle. The condition $\dot{x}(a)=0$ corresponds to the geometric statement that the tangent to the optimal curve and the tangent to the obstacle are parallel at the point of contact. This is more generally true when the integrand, like here, satisfies Legendre's condition. To illustrate this, imagine a circular obstacle between two fixed points. The shortest path between the two points must be tangent to the circle. In this case the integrand of the length functional has the form $f\left(x, y(x), y^{\prime}(x)\right)=f(x, y, z)=\sqrt{1+z^{2}}$, and the Legendre condition $\frac{\partial^{2} f}{\partial z \partial z}>0$ is satisfied.

Corollary 2.2. Consider all graphs with well-defined elastic energy such that the initial point is at the origin and the right endpoint is on the vertical line $x=1$. Assume that the initial tangent direction is horizontal and that the final tangent 
direction is vertical. The greatest lower bound of the elastic energy is positive when restricted to this collection of curves.

Proof. Parameterize each curve on $[0,1]$ so that it has constant speed equal to its length $L$, where $L>0$, and $(\dot{x}(t), \dot{y}(t))=L(\cos \theta(t), \sin \theta(t))$. The elastic energy is proportional to $E(\theta, L)=\frac{1}{L} \int_{0}^{1} \dot{\theta}^{2}(t) d t$.

The constraints are $L \int_{0}^{1} \cos \theta(t) d t=1, \theta(0)=0, \theta(1)=\pi / 2$, and the less restrictive $-\pi / 2 \leqslant \theta(t) \leqslant \pi / 2$. Let $z(t)=\cos \theta(t)$ so that $z(0)=1, z(1)=0$, and $0 \leqslant z(t) \leqslant 1$. Since $\dot{z}(t)=-\sin \theta(t) \cdot \dot{\theta}(t)$, it follows that

$$
E(\theta, L)=\int_{0}^{1} \cos \theta(t) d t \int_{0}^{1} \dot{\theta}^{2}(t) d t \geqslant \int_{0}^{1} z(t) d t \int_{0}^{1} \dot{z}^{2}(t) d t \geqslant 4 / 9 .
$$

\section{CRitical points}

In the next theorem the curves are not assumed to be graphs. The greatest lower bound is zero, but there is no global minimum. Nonetheless, there exist critical points, and each is a graph. Let $H=W_{1}^{2}[0,1]$, and consider the space $H \times \mathbb{R}^{+}$where $\mathbb{R}^{+}=\{L \in \mathbb{R}: L>0\}$. Observe that at each point the tangent space $T\left(H \times \mathbb{R}^{+}\right)$ is given by $H \times \mathbb{R}$. Write $v=\left(v_{\theta}, v_{L}\right)$ for tangent vectors $v \in H \times \mathbb{R}$, and use the Riemannian structure $\left\langle\left(v_{\theta}, v_{L}\right),\left(w_{\theta}, w_{L}\right)\right\rangle=v_{\theta}(0) w_{\theta}(0)+\int_{0}^{1} \dot{v}_{\theta}(t) \dot{w}_{\theta}(t) d t+v_{L} w_{L}$. Keep in mind that $L$ corresponds to the length of the curve and $\theta$ is the angle of its tangent. If the initial point of the curve is the origin, then its $x$-coordinate is given by $x(t)=L \int_{0}^{t} \cos \theta(s) d s$. Consider the subset $\Omega \subset H \times \mathbb{R}^{+}$given by

$$
\Omega=\left\{(\theta, L) \in H \times \mathbb{R}^{+}: \theta(0)=0, \theta(1)=\pi / 2, L \int_{0}^{1} \cos \theta(t) d t=1\right\} .
$$

Let $G, \Phi_{0}, \Phi_{1}: H \times \mathbb{R}^{+} \rightarrow \mathbb{R}$ be given by $G(\theta, L)=L \int_{0}^{1} \cos \theta(t) d t$, and $\Phi_{0}(\theta, L)=$ $\theta(0), \Phi_{1}(\theta, L)=\theta(1)$, respectively.

Lemma 3.1. The gradients have $\theta$-components

$$
\nabla G(\theta, L)_{\theta}=L\left(\int_{0}^{t} \int_{0}^{u} \sin \theta(v) d v d u-(1+t) \int_{0}^{1} \sin \theta(u) d u\right),
$$

$\nabla \Phi_{0}(\theta, L)_{\theta}=1, \nabla \Phi_{1}(\theta, L)_{\theta}=1+t$, and length components

$$
\nabla G(\theta, L)_{L}=\int_{0}^{1} \cos \theta(t) d t, \nabla \Phi_{0}(\theta, L)_{L}=\nabla \Phi_{1}(\theta, L)_{L}=0 .
$$

Proof. The derivatives are

$$
\begin{aligned}
& D G(\theta, L)\left(v_{\theta}, v_{L}\right)=v_{L} \int_{0}^{1} \cos \theta(t) d t-L \int_{0}^{1} v_{\theta}(t) \sin \theta(t) d t \\
& =v_{L} \int_{0}^{1} \cos \theta(t) d t-L v_{\theta}(1) \int_{0}^{1} \sin \theta(t) d t+L \int_{0}^{1} \dot{v}_{\theta}(t) \int_{0}^{t} \sin \theta(u) d u d t,
\end{aligned}
$$

and $D \Phi_{0}(\theta, L)\left(v_{\theta}, v_{L}\right)=v_{\theta}(0), D \Phi_{1}(\theta, L)\left(v_{\theta}, v_{L}\right)=v_{\theta}(1)$. Now it requires little more than integration by parts to verify that

$$
\langle\nabla G(\theta, L), v\rangle=\left\langle\left(\nabla G(\theta, L)_{\theta}, \nabla G(\theta, L)_{L}\right),\left(v_{\theta}, v_{L}\right)\right\rangle=D G(\theta, L)\left(v_{\theta}, v_{L}\right),
$$

and similarly for $\Phi_{0}, \Phi_{1}$. 
Lemma 3.2. The subset $\Omega \subset H \times \mathbb{R}^{+}$is a closed Riemannian submanifold of co-dimension 3.

Proof. Observe that

$$
\Omega=\left\{(\theta, L) \in H \times \mathbb{R}^{+}: G(\theta, L)=1, \Phi_{0}(\theta, L)=0, \Phi_{1}(\theta, L)=\pi / 2\right\},
$$

so the continuity of the constraints implies that $\Omega$ is closed. For $(\theta, L) \in \Omega$, assume that $\lambda \nabla G(\theta, L)+\mu_{0} \nabla \Phi_{0}(\theta, L)+\mu_{1} \nabla \Phi_{1}(\theta, L)=0$. The length component of this equation is $\lambda \cdot(1 / L)=0$, which forces $\lambda=0$. Now the $\theta$-component is reduced to $\mu_{0}+\mu_{1}(1+t)=0$. Choose $t=0$ and $t=1$ to see that $\mu_{0}=\mu_{1}=0$. It follows that the collection $\left\{\nabla G(\theta, L), \nabla \Phi_{0}(\theta, L), \nabla \Phi_{1}(\theta, L)\right\}$ is linearly independent, and that $T_{(\theta, L)} \Omega=\left\{\nabla G(\theta, L), \nabla \Phi_{0}(\theta, L), \nabla \Phi_{1}(\theta, L)\right\}^{\perp}$.

Now let $J: H \times \mathbb{R}^{+} \rightarrow \mathbb{R}$ correspond to the elastic energy by defining

$$
J(\theta, L)=\frac{1}{2 L} \int_{0}^{1} \dot{\theta}^{2}(t) d t .
$$

For each $(\theta, L) \in \Omega$, project the gradient vector field $\nabla J(\theta, L) \in H \times \mathbb{R}$ onto the tangent space $T_{(\theta, L)} \Omega \subset H \times \mathbb{R}$ and get the vector field $\nabla^{\pi} J$. A critical point is a pair $(\theta, L)$ such that $\nabla^{\pi} J(\theta, L)=(0,0)=0$. Using Jacobi's elliptic functions (sn, cn, dn as in [3]) it is possible to list every single critical point. It turns out that there is a unique critical length. Moreover, there are no 'orbit-like' critical curves. Such curves, when they exist, involve the elliptic function dn. In the case of fixed length, physical examples of orbit-like elastic curves are seen by turning one end of a wire a full turn to form a loop. Recall that

$$
\mathrm{sn}^{-1}(y, m)=\int_{0}^{y} \frac{d t}{\sqrt{1-t^{2}} \sqrt{1-m t^{2}}}
$$

yields sn, which has a continuous periodic extension to all of $\mathbb{R}$. The elliptic modulus $m$ is a number in $[0,1]$. Once $s n$ is defined, the identities $\mathrm{cn}^{2}+\mathrm{sn}^{2}=1, \mathrm{dn}^{2}+m \mathrm{sn}^{2}=$ 1 yield both cn and dn as smooth periodic even functions. The derivatives satisfy $\mathrm{sn}^{\prime}=\mathrm{cndn}, \mathrm{cn}^{\prime}=-\mathrm{sndn}$, and $\mathrm{dn}^{\prime}=-m \mathrm{cnsn}$. The following explicit antiderivative is also useful when $m>0$ :

$$
\int \operatorname{cn}(x, m) d x=\frac{1}{\sqrt{m}} \sin ^{-1}(\sqrt{m} \operatorname{sn}(x, m)) .
$$

To simplify the notation let

$$
K_{m}:=\int_{0}^{1} \frac{d t}{\sqrt{1-t^{2}} \sqrt{1-m t^{2}}}, E_{m}(t):=\int_{0}^{t} \operatorname{dn}^{2}(s, m) d s
$$

denote the two basic elliptic integrals, and put $E_{m}:=E_{m}\left(K_{m}\right)$.

Theorem 3.3. With the projected gradient given as

$$
\nabla^{\pi} J=\nabla J-\lambda \nabla G-\mu_{0} \nabla \Phi_{0}-\mu_{1} \nabla \Phi_{1},
$$

the following holds:

$$
\nabla^{\pi} J(\theta, L)=(0,0)
$$

if and only if

$$
(\theta, L)=\left(2 \sin ^{-1}\left(\sqrt{m} \operatorname{sn}\left((1+4 \eta) K_{m} t, m\right)\right), 1 /\left(\frac{2 E_{m}}{K_{m}}-1\right)\right),
$$


where the elliptic modulus $m$ satisfies $m=1 / 2$ and $\eta$ is an integer. Moreover, with $\omega=(1+4 \eta) K_{\frac{1}{2}}$ the following holds for the Lagrange multipliers: $\lambda=-\omega^{2} / L^{2}$, $\mu_{0}=-\omega \cdot \sqrt{2} / L, \mu_{1}=0$, and $J(\theta, L)=\omega^{2} / L^{2}$.

Proof. The objective has derivative

$$
D J(\theta, L)\left(v_{\theta}, v_{L}\right)=-\frac{v_{L}}{2 L^{2}} \int_{0}^{1} \dot{\theta}^{2}(t) d t+\frac{1}{L} \int_{0}^{1} \dot{v}_{\theta}(t) \dot{\theta}(t) d t
$$

and gradient $\nabla J(\theta, L)_{\theta}=(\theta(t)-\theta(0)) / L, \nabla J(\theta, L)_{L}=-J(\theta, L) / L$. The gradients simplify somewhat when restricted to $\Omega$, and there are three scalar fields, $\lambda$ and $\mu_{0}, \mu_{1}$, to ensure that the projected gradient vector field

$$
\nabla^{\pi} J=\nabla J-\lambda \nabla G-\mu_{0} \nabla \Phi_{0}-\mu_{1} \nabla \Phi_{1}
$$

is tangent to $\Omega$. This projected vector field must vanish at each critical point. With the help of Lemma 3.1 this leads to two conditions. Due to the length component one gets

$$
J(\theta, L)=-\lambda,
$$

and as an important consequence $\lambda$ is strictly negative. In the $\theta$-component the following holds when $(\theta, L) \in \Omega$ :

$$
\theta(t) / L-\lambda L\left(\int_{0}^{t} \int_{0}^{s} \sin \theta(u) d u d s-(1+t) \int_{0}^{1} \sin \theta(u) d u\right)-\mu_{0}-\mu_{1}(1+t)=0 .
$$

Set $t=0$ and deduce that

$$
\mu_{0}+\mu_{1}=\lambda L \int_{0}^{1} \sin \theta(u) d u
$$

must hold and hence

$$
\theta(t) / L-\lambda L \int_{0}^{t} \int_{0}^{s} \sin \theta(u) d u d s+t \mu_{0}=0 .
$$

Take one derivative and get

$$
\dot{\theta}(t) / L-\lambda L \int_{0}^{t} \sin \theta(u) d u+\mu_{0}=0 .
$$

It follows that the curvature at the left endpoint is equal to $-\mu_{0}$ and $\dot{\theta}(0)=-L \mu_{0}$. Observe how the integral equation expresses the first derivative in terms of two differentiable terms. Take one more derivative and get

$$
\ddot{\theta}(t)=\lambda L^{2} \sin \theta(t),
$$

the pendulum equation. A third derivative produces

$$
\dddot{\theta}(t)=\lambda L^{2} \dot{\theta}(t) \cos \theta(t) .
$$

Using the integrating factor $2 \dot{\theta}(t)$, it is seen that $\dot{\theta}^{2}(t)=-2 \lambda L^{2} \cos \theta(t)+L^{2}\left(\mu_{0}^{2}+2 \lambda\right)$ so that

$$
\dddot{\theta}(t)+\frac{\dot{\theta}^{3}(t)}{2}-L^{2}\left(\frac{\mu_{0}^{2}}{2}+\lambda\right) \dot{\theta}(t)=0 .
$$

With $\varphi(t)=\dot{\theta}(t)$, it is proved in Proposition 3.3 of [9] that to each initial condition $\varphi(0)=\varphi_{0}, \dot{\varphi}(0)=\dot{\varphi}_{0}$, there are exactly two possibilities, either

$$
\varphi(t)=\dot{\theta}(t)= \pm 2 \omega \sqrt{m} \operatorname{cn}(\omega t+\phi, m),-L^{2}\left(\frac{\mu_{0}^{2}}{2}+\lambda\right)=\omega^{2}(1-2 m)
$$


or

$$
\varphi(t)=\dot{\theta}(t)= \pm 2 \omega \operatorname{dn}(\omega t+\phi, m),-L^{2}\left(\frac{\mu_{0}^{2}}{2}+\lambda\right)=\omega^{2}(m-2)
$$

At this stage, there are therefore seven parameters $\left(L, \lambda, \mu_{0}, \mu_{1}, \omega, \phi, m\right)$ to determine. Observe that

$$
\frac{1}{2 L} \dot{\theta}^{2}(t)=-\lambda L \cos \theta(t)+L\left(\frac{\mu_{0}^{2}}{2}+\lambda\right),
$$

so integration gives $J(\theta, L)=-\lambda+L\left(\frac{\mu_{0}^{2}}{2}+\lambda\right)$. This forces $\frac{\mu_{0}^{2}}{2}+\lambda=0$ by (3.2), which in the cn-case requires $m=1 / 2$, and in the dn-case $m=2$. Since the elliptic modulus satisfies $0 \leqslant m \leqslant 1$, the dn-case is not possible. Since $\theta(0)=0$, the pendulum equation (3.4) implies that $\ddot{\theta}(0)=0$. Here $\ddot{\theta}(t)=$ $\mp 2 \omega^{2} \sqrt{m} \operatorname{sn}(\omega t+\phi, m) \operatorname{dn}(\omega t+\phi, m)$, so $\ddot{\theta}(0)=\mp 2 \omega^{2} \sqrt{m} \operatorname{sn}(\phi, m) \operatorname{dn}(\phi, m)$ forces $\phi=0, \phi=2 K_{m}$. The value $\phi=2 K_{m}$ is accounted for in $\dot{\theta}(t)= \pm 2 \omega \sqrt{m} \operatorname{cn}(\omega t, m)$. In fact, $-2 \omega \sqrt{m} \operatorname{cn}(\omega t, m)=2(-\omega) \sqrt{m} \operatorname{cn}((-\omega) t, m)$ reduces all cases to $\dot{\theta}(t)=$ $2 \omega \sqrt{m} \operatorname{cn}(\omega t, m)$, and $\theta(t)=2 \sin ^{-1}(\sqrt{m} \operatorname{sn}(\omega t, m))$ follows since $\theta(0)=0$ is required. Now the curvature condition at the left endpoint produces $L \mu_{0}=-2 \omega \sqrt{m}$. The pendulum equation (3.4) and

$$
\sin \theta(t)=2 \sqrt{m} \operatorname{sn}(\omega t, m) \operatorname{dn}(\omega t, m)
$$

forces $\lambda L^{2}=-\omega^{2}$. The constraint $\theta(1)=\pi / 2$ corresponds to $\operatorname{sn}(\omega, 1 / 2)=1$ and $\omega=(1+4 \eta) K_{m}$ for some integer $\eta$. The constraint $L \int_{0}^{1} \cos \theta(t) d t=1$ determines L. Observe that

$$
\cos \left(2 \sin ^{-1}(\sqrt{m} \operatorname{sn}(\omega t, m))\right)=1-2 m \operatorname{sn}^{2}(\omega t, m)=2 \operatorname{dn}^{2}(\omega t, m)-1,
$$

so the constraint's integral is expressed as the elliptic integral $E$, specifically

$$
\frac{2 E_{m}(\omega)}{\omega}-1=\frac{1}{L}
$$

Now $E_{m}\left((1+4 \eta) K_{m}\right)=(1+4 \eta) E_{m}$, so $L$ is a unique value independent of $\eta$. The value of $\mu_{1}$ is expressed in terms of the present data by using (3.3). The key calculation is

$$
\int_{0}^{1} \sin \theta(t) d t=[(-2 \sqrt{m} / \omega) \operatorname{cn}(\omega t, m)]_{0}^{1}=\sqrt{2} / \omega .
$$

To finish the proof it is necessary to verify that all constraints are satisfied, and that $\nabla^{\pi} J(\theta, L)=(0,0)$. When $\theta(t)=2 \sin ^{-1}\left(\sqrt{m} \operatorname{sn}\left((1+4 \eta) K_{m} t, m\right)\right)$, it is clear that $\theta(0)=0$. With the elliptic modulus $m=1 / 2$, it is also true that $\theta(1)=\pi / 2$. The constraint $L \int_{0}^{1} \cos \theta(t) d t=1$ is satisfied since

$$
\int_{0}^{1} \cos \theta(t) d t=2 E_{m} / K_{m}-1
$$

and $L$ is chosen so this is equal to $1 / L$. Finally, $\theta$ satisfies $\ddot{\theta}(t)=\lambda L^{2} \sin \theta(t)$, and hence

$$
\dot{\theta}(t) / L-\lambda L \int_{0}^{t} \sin \theta(u) d u+\mu_{0}=0,
$$

where the value of the constant of integration is picked so that $\dot{\theta}(0) / L=-\mu_{0}$. One more integration leads to $\theta(t) / L-\lambda L \int_{0}^{t} \int_{0}^{s} \sin \theta(u) d u d s+t \mu_{0}=0$, where the value of the constant of integration ensures $\theta(0)=0$. The choice of $\mu_{1}$ shows that $\left(\nabla^{\pi} J(\theta, L)\right)_{\theta}=0$, and $J(\theta, L)=-\lambda$ guarantees that $\left(\nabla^{\pi} J(\theta, L)\right)_{L}=0$. The last 
step is to verify that $m=1 / 2$ and $\omega=(1+4 \eta) K_{m}$ produces $L>1$, and this is indeed the case because the length $L$ is approximately 2.18844 .

Remark 1 . The elastic energy represented by $J$ for the critical curve with $\eta=0$ is approximately 0.71777 .

Remark 2. The greatest lower bound for $J$ in this problem is zero. To see this, build a curve using a straight segment followed by a counterclockwise semi-circle, and end the curve by a clockwise quarter circle. As the radii of the two circular arcs increase to infinity, the elastic energy tends to zero.

Remark 3 . The $y$-coordinate at the right endpoint of the critical curve when $\eta=0$ is approximately 1.66925; see Figure 1.

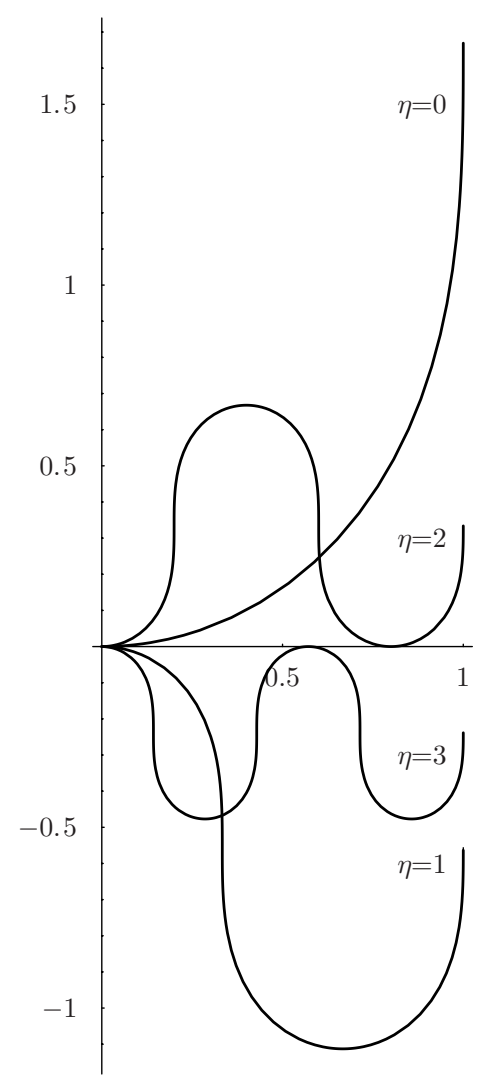

Figure 1. The critical curves with $\eta=0,1,2,3$.

\section{Fixed Length}

Theorem 4.1. Write $H=W_{1}^{2}[0,1]$ and let $E: H \rightarrow \mathbb{R}$ be given by $E(\theta)=$ $\int_{0}^{1} \dot{\theta}^{2}(t) d t$. Consider $\Omega_{c} \subset H$ defined by

$$
\Omega_{c}=\left\{\theta \in H: \theta(0)=0, \theta(1)=\pi / 2, \int_{0}^{1} \cos \theta(t) d t=c,|\theta(t)| \leqslant \pi / 2\right\} .
$$


For each $c$, such that $0<c<1$, there is a function $\hat{\theta}_{c} \in \Omega_{c}$ with $E\left(\hat{\theta}_{c}\right) \leqslant E\left(\theta_{c}\right)$ for all $\theta_{c} \in \Omega_{c}$.

Recall that the constraint $L \int_{0}^{1} \cos \theta(t) d t=1$ corresponds to the fact that the curve has a horizontal distance of one unit between the endpoints. It follows that $c=1 / L$.

Proof. The key observation is the following fact:

- $\Omega_{c}$ is weakly sequentially closed in $W_{1}^{2}[0,1]$.

To substantiate this, note that, for $\theta \in \Omega_{c}, E(\theta)$ represents the squared norm of $\theta$. By the compact version of the Sobolev embedding theorem ((8), p. 168 of [1]), the injection $W_{1}^{2}[0,1] \rightarrow C^{\lambda}[0,1], \quad 0<\lambda<1 / 2$, is compact. Here, $\lambda$ denotes the usual Hölder index. In particular, the injection is compact into $C[0,1]$. Now if $\left\{\theta_{k}\right\} \subset \Omega_{c}$ converges weakly to $\theta$ in $W_{1}^{2}[0,1]$, we conclude by the above that $\left\{\theta_{k}\right\}$ converges uniformly to $\theta$; this is a consequence of the property of compact mappings, which map weakly convergent sequences onto convergent sequences. The uniform convergence, in turn, implies that each of the defining properties for $\Omega_{c}$ is transmitted to $\theta$, i.e., $\theta \in \Omega_{c}$. Now suppose $\left\{\theta_{k}\right\} \subset \Omega_{c}$ is any minimizing sequence, so that $E\left(\theta_{k}\right) \rightarrow \inf _{\Omega_{c}} E$. The sequence $\left\{\theta_{k}\right\}$ is bounded in $W_{1}^{2}[0,1]$. By the weak compactness of closed balls in this space, there is a weakly convergent subsequence, $\theta_{k_{j}} \rightarrow \hat{\theta}_{c}, j \rightarrow \infty$. The weak limit satisfies $\hat{\theta}_{c} \in \Omega_{c}$ since $\Omega_{c}$ is weakly sequentially closed. We may use the lower semicontinuity of the norm (Theorem 1, p. 120 of [12]) with respect to weak convergence to deduce that $E\left(\hat{\theta}_{c}\right) \leqslant \liminf _{j \rightarrow \infty} E\left(\theta_{k_{j}}\right)=\inf _{\Omega_{c}} E$.

Remark 1. The previous reasoning is still applicable when the constraint $|\theta(t)| \leqslant$ $\pi / 2$ is absent.

Remark 2. It is essential that the isoperimetric constraint does not involve the derivative of the functions in the domain.

To state the next result, let $F, G, \phi_{0}, \phi_{1}: W_{1}^{2}[0,1] \rightarrow \mathbb{R}$ be given by $F(\theta)=$ $\frac{1}{2} \int_{0}^{1} \dot{\theta}^{2}(t) d t, G(\theta)=\int_{0}^{1} \cos \theta(t) d t, \phi_{0}(\theta)=\theta(0), \phi_{1}(\theta)=\theta(1)$. Analogous to the case of variable length (cf. Lemma 3.1 and Theorem 3.3), the gradients are

$$
\begin{gathered}
\nabla F(\theta)=\theta(t)-\theta(0), \nabla \phi_{0}(\theta)=1, \nabla \phi_{1}(\theta)=1+t, \\
\nabla G(\theta)=\int_{0}^{t} \int_{0}^{s} \sin \theta(u) d u d s-(1+t) \int_{0}^{1} \sin \theta(s) d s .
\end{gathered}
$$

As before, let $\lambda, \mu_{0}, \mu_{1}$ be real scalar fields so that

$$
\nabla^{\pi} F=\nabla F-\lambda \nabla G-\mu_{0} \nabla \phi_{0}-\mu_{1} \nabla \phi_{1}
$$

is the projection onto the tangent space of the constrained set $G(\theta)=c, \phi_{0}(\theta)=0$, $\phi_{1}(\theta)=\pi / 2$, where $0<c<1$. A reasoning similar to the case of variable length shows that this constrained set is a closed Riemannian submanifold of co-dimension 3 .

Theorem 4.2. A function $\theta \in \Omega_{c}$ of minimal elastic energy must be given by one of the following five cases. Partition the interval $(0,1)$ as

$$
\left(0, c_{\frac{1}{2}}\right) \cup\left[c_{\frac{1}{2}}, c_{1}\right] \cup\left(c_{1}, \frac{2}{\pi}\right) \cup\left\{\frac{2}{\pi}\right\} \cup\left(\frac{2}{\pi}, 1\right),
$$


where $c_{\frac{1}{2}}=\frac{2 E_{\frac{1}{2}}}{K_{\frac{1}{2}}}-1 \approx 0.456947, c_{1}=\frac{2 E_{1}\left(\tanh ^{-1}\left(\frac{1}{\sqrt{2}}\right)\right)}{\tanh ^{-1}\left(\frac{1}{\sqrt{2}}\right)}-1 \approx 0.604556, \frac{2}{\pi} \approx 0.63662$.

When $c \in\left[c_{\frac{1}{2}}, c_{1}\right], \theta(t)=2 \sin ^{-1}(\sqrt{m} \operatorname{sn}(\omega t, m))$ for some $1 / 2 \leqslant m \leqslant 1$, $\omega$ such that

$$
\begin{aligned}
& \sqrt{m} \operatorname{sn}(\omega, m)=1 / \sqrt{2}, \\
& \frac{2 E_{m}(\omega)}{\omega}-1=c,
\end{aligned}
$$

and $\lambda=-\omega^{2}, \mu_{0}=-2 \omega \sqrt{m}, \mu_{1}=2 \omega \sqrt{m-\frac{1}{2}}$.

When $c \in\left(c_{1}, \frac{2}{\pi}\right), \theta(t)=2 \sin ^{-1}(\operatorname{sn}(\omega t, m))$ for some $0<m<1$, $\omega$ such that

$$
\begin{aligned}
& \operatorname{sn}(\omega, m)=1 / \sqrt{2}, \\
& \frac{2 E_{m}(\omega)}{\omega m}+1-\frac{2}{m}=c,
\end{aligned}
$$

and $\lambda=-\omega^{2} m, \mu_{0}=-2 \omega, \mu_{1}=2 \omega \sqrt{1-\frac{m}{2}}$.

When $c \in\left(\frac{2}{\pi}, 1\right), \theta(t)=2 \sin ^{-1}\left(\operatorname{sn}\left(\omega t-K_{m}, m\right)\right)+\pi$ for some $0<m<1$, $\omega$ such that

$$
\begin{aligned}
& \operatorname{sn}\left(\omega-K_{m}, m\right)=-1 / \sqrt{2}, \\
& \frac{2}{m}-1-\frac{2}{\omega m}\left(E_{m}\left(\omega-K_{m}\right)-E_{m}\left(-K_{m}\right)\right)=c,
\end{aligned}
$$

and $\lambda=\omega^{2} m, \mu_{0}=-2 \omega \sqrt{1-m}, \mu_{1}=2 \omega \sqrt{1-\frac{m}{2}}$.

When $c \in\left(0, c_{\frac{1}{2}}\right)$,

$$
\theta(t)=\left\{\begin{array}{l}
2 \sin ^{-1}\left(\sqrt{m} \operatorname{sn}\left(K_{m} t / a, m\right)\right), 0<t \leqslant a \\
\pi / 2, a<t \leqslant 1
\end{array}\right.
$$

where $m=1 / 2$ and $0<a<1$ is such that $a\left(\frac{2 E_{m}}{K_{m}}-1\right)=c$.

When $c=2 / \pi$, then $\theta(t)=\pi t / 2$, and $\lambda=0, \mu_{0}=-\pi / 2, \mu_{1}=\pi / 2$. The corresponding curve is a quarter of the unit circle.

Moreover, the projected gradient vector field $\nabla^{\pi} F$ vanishes when $c \in\left[c_{\frac{1}{2}}, 1\right)$ if and only if $\theta$ is given as indicated by the corresponding case.

Finally, the elastic energy $c F(\theta)$ is given by $c F(\theta)=c \omega^{2}(c+2 m-1)$ on $\left[c_{\frac{1}{2}}, c_{1}\right]$, and $c F(\theta)=c \omega^{2}(m c+2-m)$ on $\left[c_{1}, 1\right)$. The energy, as a function of $c$, is constant on $\left(0, c_{\frac{1}{2}}\right]$ with a value, calculated by using $c=c_{\frac{1}{2}}, m=1 / 2, \omega=K_{\frac{1}{2}}$, approximately equal to 0.71777. The energy at $c_{1}$ is approximately 0.75355 with $m=1$ and $\omega=\tanh ^{-1}(1 / \sqrt{2})$. The energy of a quarter of a circle is $\pi / 4 \approx 0.785398$.

Proof. The proof is divided into two parts. In the first part the analysis proceeds without concern for the constraint $|\theta(t)| \leqslant \pi / 2$. The second part resolves the issues due to the inclusion of the constraint $|\theta(t)| \leqslant \pi / 2$.

Part 1 of the proof. Temporarily postpone the consideration of the constraint $|\theta(t)| \leqslant \pi / 2$. The projected gradient vector field vanishes if and only if

$$
\nabla^{\pi} F(\theta)=\nabla F(\theta)-\lambda \nabla G(\theta)-\mu_{0} \nabla \phi_{0}(\theta)-\mu_{1} \nabla \phi_{1}(\theta)=0,
$$

which takes the form

$$
\theta(t)-\lambda\left(\int_{0}^{t} \int_{0}^{s} \sin \theta(u) d u d s-(1+t) \int_{0}^{1} \sin \theta(s) d s\right)-\mu_{0}-\mu_{1}(1+t)=0 .
$$


Let $t=0$ and deduce that

$$
\lambda \int_{0}^{1} \sin \theta(s) d s=\mu_{0}+\mu_{1},
$$

so the projected vector field equation simplifies to

$$
\theta(t)-\lambda \int_{0}^{t} \int_{0}^{s} \sin \theta(u) d u d s+t \mu_{0}=0 .
$$

Differentiate and get

$$
\dot{\theta}(t)-\lambda \int_{0}^{t} \sin \theta(s) d s+\mu_{0}=0 .
$$

Put $t=0$ and observe that

$$
\mu_{0}=-\dot{\theta}(0) .
$$

Differentiate one more time and get the fact that the pendulum equation must be satisfied in the form

$$
\ddot{\theta}(t)=\lambda \sin \theta(t) .
$$

A third derivative yields

$$
\dddot{\theta}(t)=\lambda \dot{\theta}(t) \cos \theta(t),
$$

and the integrating factor $2 \dot{\theta}(t)$ produces $\dot{\theta}^{2}(t)=-2 \lambda \cos \theta(t)+\mu_{0}^{2}+2 \lambda$. When these last two equations are combined, one gets

$$
\dddot{\theta}(t)+\frac{\dot{\theta}^{3}(t)}{2}-\left(\frac{\mu_{0}^{2}}{2}+\lambda\right) \dot{\theta}(t)=0 .
$$

As before, by Proposition 3.3 in [9] there are only two possible solutions. The first is given by

$$
\dot{\theta}_{a}(t)= \pm 2 \omega \sqrt{m} \operatorname{cn}(\omega t+\phi, m),
$$

with $-\left(\frac{\mu_{0}^{2}}{2}+\lambda\right)=\omega^{2}(1-2 m)$. The second possibility is given by

$$
\dot{\theta}_{b}(t)= \pm 2 \omega \operatorname{dn}(\omega t+\phi, m)
$$

with $-\left(\frac{\mu_{0}^{2}}{2}+\lambda\right)=\omega^{2}(m-2)$.

Part 1 (the cn-case). In the first case the second derivative is

$$
\ddot{\theta}_{a}(t)=\mp 2 \omega^{2} \sqrt{m} \operatorname{sn}(\omega t+\phi, m) \operatorname{dn}(\omega t+\phi, m) .
$$

Observe that the pendulum equation (4.6) implies that

$$
\ddot{\theta}_{a}(0)=\mp 2 \omega^{2} \sqrt{m} \operatorname{sn}(\phi, m) \operatorname{dn}(\phi, m)=0,
$$

so either $\phi=0, \phi=2 K_{m}$, or $m=0$. The case $m=0$ leads to $\dot{\theta}_{a}(t)=0$, which is inconsistent with the boundary conditions. Moreover, the case $\phi=2 K_{m}$ is covered by $\dot{\theta}_{a}(t)= \pm 2 \omega \sqrt{m} \operatorname{cn}(\omega t, m)$.

Observe that $\mathrm{cn}$ is even, so if needed replace $\omega$ by $-\omega$ to see that $\dot{\theta}_{a}(t)=$ $2 \omega \sqrt{m} \operatorname{cn}(\omega t, m)$ is no less general. Since $\theta_{a}(0)=0$, it must be that $\theta_{a}(t)=$ $2 \sin ^{-1}(\sqrt{m} \operatorname{sn}(\omega t, m))$, and since $\sin \theta_{a}(t)=2 \sqrt{m} \operatorname{sn}(\omega t, m) \operatorname{dn}(\omega t, m)$, it follows that $\lambda=-\omega^{2}$ again by (4.6). Let $t=0$ and observe that the formula (4.5) for $\mu_{0}$ produces $\mu_{0}=-2 \omega \sqrt{m}$. The formula (4.4) for $\mu_{1}$ involves the integral

$$
\int_{0}^{1} \sin \theta_{a}(t) d t=-\frac{2 \sqrt{m}}{\omega}[\operatorname{cn}(\omega t, m)]_{0}^{1}=\frac{2 \sqrt{m}}{\omega}(1-\operatorname{cn}(\omega, m)) .
$$


The constraint $\theta(1)=\pi / 2$ translates into $2 \sin ^{-1}(\sqrt{m} \operatorname{sn}(\omega, \mathrm{m}))=\pi / 2$, which simplifies to $\sqrt{m} \operatorname{sn}(\omega, \mathrm{m})=1 / \sqrt{2}$ and necessitates $m \geqslant 1 / 2$. Put things together and conclude that

$$
\mu_{1}=-2 \omega \sqrt{m}(1-\operatorname{cn}(\omega, m))-\mu_{0}=2 \omega \sqrt{m} \sqrt{1-\frac{1}{2 m}}=2 \omega \sqrt{m-\frac{1}{2}} .
$$

The constraint $\int_{0}^{1} \cos \theta_{a}(t) d t=c$ has

$$
\cos \theta_{a}(t)=1-2 m \operatorname{sn}^{2}(\omega t, m)=2 \operatorname{dn}^{2}(\omega t, m)-1,
$$

so in terms of the elliptic integral $E_{m}$ the condition is $\frac{2 E_{m}(\omega)}{\omega}-1=c$.

Part 2 (the two dn-cases). In the second case $\dot{\theta}_{b}(t)= \pm 2 \omega \operatorname{dn}(\omega t+\phi, m)$; the second derivative is $\ddot{\theta}_{b}(t)=\mp 2 \omega^{2} m \operatorname{sn}(\omega t+\phi, m) \operatorname{cn}(\omega t+\phi, m)$. The pendulum equation implies that $\ddot{\theta}_{b}(0)=\mp 2 \omega^{2} m \operatorname{sn}(\phi, m) \operatorname{cn}(\phi, m)=0$, so either $\phi=0, \phi=$ $2 K_{m}, \phi=-K_{m}, \phi=K_{m}$, or $m=0$. The case $m=0$ leads to $\theta_{b}(t)=\pi t / 2$, and the pendulum equation forces $\lambda=0$. It follows that $\mu_{0}=-\pi / 2, \mu_{1}=\pi / 2$, and it must be that $c=2 / \pi$. For all other $c$ it is necessary to have $m>0$. The cases $\phi=2 K_{m}$, $\phi=K_{m}$ are included in $\dot{\theta}_{b}(t)= \pm 2 \omega \operatorname{dn}(\omega t, m), \dot{\theta}_{b}(t)= \pm 2 \omega \operatorname{dn}\left(\omega t-K_{m}, m\right)$, respectively. Observe that $\mathrm{dn}$ is even, so if needed replace $\omega$ by $-\omega$ to see that $\dot{\theta}_{b}(t)=2 \omega \operatorname{dn}(\omega t, m), \dot{\theta}_{b}(t)=2 \omega \operatorname{dn}\left(\omega t-K_{m}, m\right)$ are no less general. Since $\theta_{b}(0)=$ 0 , it must be that $\theta_{b}(t)=2 \sin ^{-1}(\operatorname{sn}(\omega t, m))$ or $\theta_{b}(t)=2 \sin ^{-1}\left(\operatorname{sn}\left(\omega t-K_{m}, m\right)\right)+\pi$. In the first case, $\sin \theta_{b}(t)=2 \operatorname{sn}(\omega t, m) \operatorname{cn}(\omega t, m)$, and it follows that $\lambda=-\omega^{2} m$. In the second case $\sin \theta_{b}(t)=-2 \operatorname{sn}\left(\omega t-K_{m}, m\right) \operatorname{cn}\left(\omega t-K_{m}, m\right)$ and this time $\lambda=\omega^{2} m$. Let $t=0$ and observe that the formula for $\mu_{0}$ produces $\mu_{0}=-2 \omega$ and $\mu_{0}=-2 \omega \sqrt{1-m}$, respectively. The formulas for $\mu_{1}$ involve the integrals

$$
\begin{gathered}
\int_{0}^{1} \sin \theta_{b}(t) d t=-\frac{2}{\omega m}[\operatorname{dn}(\omega t, m)]_{0}^{1}=\frac{2}{\omega m}(1-\operatorname{dn}(\omega, m)), \\
\int_{0}^{1} \sin \theta_{b}(t) d t=\frac{2}{\omega m}\left[\operatorname{dn}\left(\omega t-K_{m}, m\right)\right]_{0}^{1}=\frac{2}{\omega m}\left(\operatorname{dn}\left(\omega-K_{m}, m\right)-\sqrt{1-m}\right) .
\end{gathered}
$$

The constraint $\theta(1)=\pi / 2$ translates into

$$
2 \sin ^{-1}(\operatorname{sn}(\omega, \mathrm{m}))=\pi / 2,2 \sin ^{-1}\left(\operatorname{sn}\left(\omega-K_{m}, m\right)\right)+\pi=\pi / 2,
$$

which simplify to

$$
\operatorname{sn}(\omega, \mathrm{m})=1 / \sqrt{2}, \operatorname{sn}\left(\omega-K_{m}, m\right)=-1 / \sqrt{2} .
$$

Put things together and conclude that in both cases $\mu_{1}=2 \omega \sqrt{1-\frac{m}{2}}$. The constraint $\int_{0}^{1} \cos \theta_{b}(t) d t=c$ has

$$
\begin{gathered}
\cos \theta_{b}(t)=1-2 \operatorname{sn}^{2}(\omega t, m)=\frac{2}{m} \operatorname{dn}^{2}(\omega t, m)+1-\frac{2}{m}, \\
\cos \theta_{b}(t)=2 \operatorname{sn}^{2}\left(\omega t-K_{m}, m\right)-1=\frac{2}{m}-1-\frac{2}{m} \operatorname{dn}^{2}\left(\omega t-K_{m}, m\right) .
\end{gathered}
$$

In terms of the elliptic integral $E_{m}$ the conditions are

$$
\frac{2 E_{m}(\omega)}{\omega m}+1-\frac{2}{m}=c, \frac{2}{m}-1-\frac{2}{\omega m}\left(E_{m}\left(\omega-K_{m}\right)-E_{m}\left(-K_{m}\right)\right)=c,
$$

respectively. At this stage, with the constraint $|\theta(t)| \leqslant \pi / 2$ absent, the form of all existing solutions have been identified. 
Part 2 of the proof. In the presence of the constraint $|\theta(t)| \leqslant \pi / 2$, it is necessary to apply Pontrjagin's maximum principle to the case of phase constraints. The notation conforms to Theorem 1 , page 234, in [6]. Let

$$
\begin{aligned}
& x_{1}(t)=\theta(t), \\
& x_{2}(t)=\int_{0}^{t} \cos \theta(s) d s,
\end{aligned}
$$

with endpoint constraints

$$
\begin{aligned}
& x_{1}(0)=0, \quad x_{1}(1)=\pi / 2, \\
& x_{2}(0)=0, \quad x_{2}(1)=c .
\end{aligned}
$$

There is one control variable $u$ and the following holds

$$
\begin{aligned}
& \dot{x}_{1}(t)=u(t), \\
& \dot{x}_{2}(t)=\cos x_{1}(t) .
\end{aligned}
$$

The functional is given by

$$
F\left(x_{1}(\cdot), x_{2}(\cdot), u(\cdot)\right)=\frac{1}{2} \int_{0}^{1} u^{2}(t) d t .
$$

Define the functions

$$
\begin{gathered}
f\left(t, x_{1}, x_{2}, u\right)=\frac{u^{2}}{2}, \\
\varphi_{1}\left(t, x_{1}, x_{2}, u\right)=u, \varphi_{2}\left(t, x_{1}, x_{2}, u\right)=\cos x_{1}, \\
g_{1}\left(t, x_{1}(t), x_{2}(t)\right)=x_{1}(t)-\frac{\pi}{2} \leqslant 0, g_{2}\left(t, x_{1}(t), x_{2}(t)\right)=-x_{1}(t)-\frac{\pi}{2} \leqslant 0, \\
h_{0}\left(x_{1}(0), x_{2}(0)\right)=\left(x_{1}(0), x_{2}(0)\right)=(0,0), \\
h_{1}\left(x_{1}(1), x_{2}(1)\right)=\left(x_{1}(1)-\frac{\pi}{2}, x_{2}(1)-c\right)=(0,0) .
\end{gathered}
$$

The Pontrjagin function is

$$
P\left(t, x_{1}, x_{2}, u, p_{1}, p_{2}, \lambda_{0}\right)=p_{1}(t) u(t)+p_{2}(t) \cos x_{1}(t)-\lambda_{0} \frac{u^{2}(t)}{2} .
$$

The Hamiltonian is

$$
H\left(t, x_{1}, x_{2}, p_{1}, p_{2}, \lambda_{0}\right)=\frac{p_{1}^{2}(t)}{\lambda_{0}}+p_{2}(t) \cos x_{1}(t) .
$$

If $\left(\hat{x}_{1}(\cdot), \hat{x}_{2}(\cdot), \hat{u}(\cdot)\right)$ is optimal, then $\lambda_{0} \hat{u}(t)=p_{1}(t)$ for almost all $t \in[0,1]$. The ' $x$-gradient' of the Pontrjagin function is $P_{x}=\left(-p_{2} \sin x_{1}, 0\right)$. At each optimal solution there is a multiplier $\left(l_{1}^{1}, l_{1}^{2}\right)$ and a pair of regular measures $\mu_{1}, \mu_{2}$ supported on $T_{i}=\left\{t: g_{i}\left(t, x_{1}(t), x_{2}(t)\right)=0\right\}$, with $i=1,2$, respectively, satisfying the vector equation

$$
\begin{aligned}
\left(p_{1}(t), p_{2}(t)\right)= & -\left(l_{1}^{1}, l_{1}^{2}\right)+\int_{t}^{1}\left(-p_{2}(\tau) \sin \hat{x}_{1}(\tau), 0\right) d \tau \\
& -\int_{t}^{1}(1,0) d \mu_{1}-\int_{t}^{1}(-1,0) d \mu_{2} .
\end{aligned}
$$

The second coordinate reduces to $p_{2}(t)=-l_{1}^{2}$. This implies that

$$
p_{1}(t)=-l_{1}^{1}+l_{1}^{2} \int_{t}^{1} \sin \hat{x}_{1}(\tau) d \tau-\int_{t}^{1} d \mu_{1}+\int_{t}^{1} d \mu_{2} .
$$


It must be that an optimal curve increases, because if $\theta(t)<0$, then due to continuity there is some $s>t$ such that $\theta(s)=0$, and the elastic energy is reduced if $\theta$ is replaced by a function that vanishes on $[0, s]$. To avoid changing the length of the curve, attach a vertical straight segment of the appropriate length at the right endpoint. From this it follows that an optimal solution will never satisfy $\theta(t)=-\pi / 2$, so the last integral in (4.7) is zero. The equation takes on the form

$$
\lambda_{0} \dot{\theta}(t)=p_{1}(t)=-l_{1}^{1}+l_{1}^{2} \int_{t}^{1} \sin \theta(\tau) d \tau-\int_{t}^{1} d \mu_{1} .
$$

If $\theta\left(t^{\prime}\right)=\pi / 2$ and $\theta(t)<\pi / 2$ for some $0<t^{\prime}<t \leqslant 1$, then $\theta$ is not optimal. To see this, consider the following construction. Start with the initial piece and observe that $\theta(0)=0$. Attach a horizontal straight segment to the left endpoint of the curve and adjust the length of this segment so that the new curve can be placed with the left endpoint at the origin and the right endpoint on the vertical line $x=1$. The length of this new curve is less than the length of the whole original curve. To finish the construction, attach a straight vertical line segment at the right endpoint and adjust its length so that the new curve has the same length as the whole original curve. The elastic energy of this new curve is less than the original curve.

Recall that when the constraint $|\theta(t)| \leqslant \pi / 2$ is absent there is a global minimum for each $0<c<1$. An examination of the level curves generated by the systems (4.1), (4.2), (4.3), and different values for $c$, reveals that when $0<c<c_{\frac{1}{2}}$ the global minimum violates the constraint $|\theta(t)| \leqslant \pi / 2$. It follows that for $0<c<c_{\frac{1}{2}}$ the global minimum must have $\theta\left(t^{\prime}\right)=\pi / 2$ for some $0<t^{\prime}<1$ when $|\theta(t)| \leqslant \pi / 2$ is imposed. Hence there is a $t_{0} \in(0,1)$ so that $\theta(t)=\pi / 2$ for $t \geqslant t_{0}$, and $\theta(t)<\pi / 2$ for $t<t_{0}$. If $t<t_{0}$, then $\int_{t}^{1} d \mu_{1}=\int_{t_{0}}^{1} d \mu_{1}=\mu_{1}\left(\left[t_{0}, 1\right]\right)$, and

$$
\lambda_{0} \ddot{\theta}(t)=-l_{1}^{2} \sin \theta(t),
$$

by (4.8). If $\lambda_{0} \neq 0$, then this is the pendulum equation and the solution for $t<t_{0}$ is given by elliptic functions. The curve has a vertical line segment at the right endpoint. If $\lambda_{0}=0$, then $\theta(1)=\pi / 2$ forces $l_{1}^{2}=0$ by (4.9). Since this implies $0=p_{1}(t)=-l_{1}^{1}-\int_{t}^{1} d \mu_{1}$, either $l_{1}^{1}=0$ and $\mu_{1}=0$, or the support of $\mu_{1}$ is the single point $t=1$. The first possibility forces all multipliers to be zero, which is not allowed. The second possibility is due to the redundancy of the phase constraint $\theta(t) \leqslant \pi / 2$ and the endpoint constraint $\theta(1)=\pi / 2$ when $t=1$. Since $\theta\left(t^{\prime}\right)=\pi / 2$ for some $0<t^{\prime}<1$, the second case is not relevant here.

The derivative of an optimal function must be bounded; see www.math.niu. $\mathrm{edu} / \sim$ alinner.

The three distinct systems of nonlinear equations (4.1), (4.2), and (4.3) imply the following. Minimal energy curves of relatively short length $L$, so that $c=1 / L \in$ $\left(\frac{2}{\pi}, 1\right)$, correspond to solutions of the system

$$
\operatorname{sn}\left(\omega-K_{m}, m\right)=-1 / \sqrt{2}, \quad \frac{2}{m}-1-\frac{2}{\omega m}\left(E_{m}\left(\omega-K_{m}\right)-E_{m}\left(-K_{m}\right)\right)=c .
$$

The parameter $c$ appears only in the second equation in each system (4.1), (4.2), and (4.3). Moreover, the variable $\omega$ can be expressed in terms of the elliptic modulus $m$ and $\mathrm{sn}^{-1}$ using the first equation in (4.1), (4.2), and (4.3). Replace $\omega$ in the second equation of the systems (4.1), (4.2), and (4.3). This leads to functions of one variable. Here are some properties. The elliptic modulus satisfies $m \rightarrow 1^{-}$ as $c \rightarrow 1^{-}$, and $m \rightarrow 0^{+}$as $c \rightarrow(2 / \pi)^{+}$; see Figure 2 for an illustration of these 
properties. A transition takes place at the value $c=2 / \pi$ when the solution curve is a quarter of a circle. The multiplier $\lambda$ changes sign from positive to negative so both $\lambda$ and $m$ are zero at the transition. As the length increases and $c \in\left(c_{1}, \frac{2}{\pi}\right)$, solutions correspond to the system

$$
\operatorname{sn}(\omega, m)=1 / \sqrt{2}, \quad \frac{2 E_{m}(\omega)}{\omega m}+1-\frac{2}{m}=c .
$$

This time $m \rightarrow 1^{-}$as $c \rightarrow c_{1}^{+}$. Observe how narrow the interval $\left(c_{1}, \frac{2}{\pi}\right)$ is as it deals with lengths between approximately 1.571 and 1.654; see Figure 3. The transition at $c_{1}$ yields hyperbolic functions and is included as a limit case of the elliptic functions generated by $c$ in the interval $\left[c_{\frac{1}{2}}, c_{1}\right]$. In this case the curves correspond to solutions of

$$
\sqrt{m} \operatorname{sn}(\omega, m)=1 / \sqrt{2}, \quad \frac{2 E_{m}(\omega)}{\omega}-1=c .
$$

The first equation implies that $m \geqslant 1 / 2$. Recall that, as $c$ decreases, the elastic energy decreases, and $m=1 / 2$ when $c=c_{\frac{1}{2}}$. This case is illustrated in Figure 4 , where the lowest branch of the level curve $\sqrt{m} \operatorname{sn}(\omega, m)=1 / \sqrt{2}$ has a vertical tangent line at this point. Observe how the two level curves intersect at $\left(\frac{1}{2}, K_{\frac{1}{2}}\right) \approx$ $(0.5,1.85407)$. Points on the level curve $\sqrt{m} \operatorname{sn}(\omega, m)=1 / \sqrt{2}$ above this point of intersection lead to a violation of the constraint $|\theta(t)| \leqslant \pi / 2$. This exceptional point is the 'last' point that corresponds to a solution of the three nonlinear systems (4.1), (4.2), and (4.3). The other two dashed curves illustrate the intersection of the two level curves when $c=c_{1}$ so $m=1$, and also for some 'generic' value of $c \in\left(c_{\frac{1}{2}}, c_{1}\right)$ between the two extremes. Note that no curve encountered so far has had any vertical line segments. As $c \in\left(0, c_{\frac{1}{2}}\right)$ the Pontrjagin argument becomes operative, and a vertical line segment of appropriate length is attached at the right endpoint.

The two energy formulas, $c F(\theta)=c \omega^{2}(c+2 m-1)$ on $\left[c_{\frac{1}{2}}, c_{1}\right]$, and $c F(\theta)=$ $c \omega^{2}(m c+2-m)$ on $\left[c_{1}, 1\right)$, come about by integrating the equation

$$
\dot{\theta}^{2}(t)=-2 \lambda \cos \theta(t)+\mu_{0}^{2}+2 \lambda,
$$

and replacing the multipliers by their values in the cn and dn cases, respectively.

Finally, when $c \in\left[c_{\frac{1}{2}}, 1\right)$, explicit integrations and evaluations show that solutions produced by the three systems (4.1), (4.2), and (4.3), all have the property that $\nabla^{\pi} \mathrm{F}(\theta)=0$. To illustrate this, let $\theta(t)=2 \sin ^{-1}(\sqrt{m} \operatorname{sn}(\omega t, \mathrm{~m}))$, where

$$
\sqrt{m} \operatorname{sn}(\omega, m)=1 / \sqrt{2}, \frac{2 E_{m}(\omega)}{\omega}-1=c .
$$

It is immediate that $\theta(0)=0, \theta(1)=\pi / 2$, and $\int_{0}^{1} \cos \theta(t) d t=c$. Let $\lambda=-\omega^{2}, \mu_{0}=$ $-2 \omega \sqrt{m}, \mu_{1}=2 \omega \sqrt{1-\frac{m}{2}}$. The previous calculations show that $\ddot{\theta}(t)=\lambda \sin \theta(t)$. Integrate this equation and choose the constant of integration to be $\mu_{0}$ so that $\dot{\theta}(t)-\lambda \int_{0}^{t} \sin \theta(s) d s+\mu_{0}=0$. One more integration yields

$$
\theta(t)-\lambda \int_{0}^{t} \int_{0}^{s} \sin \theta(u) d u d s+\mu_{0} t=0
$$




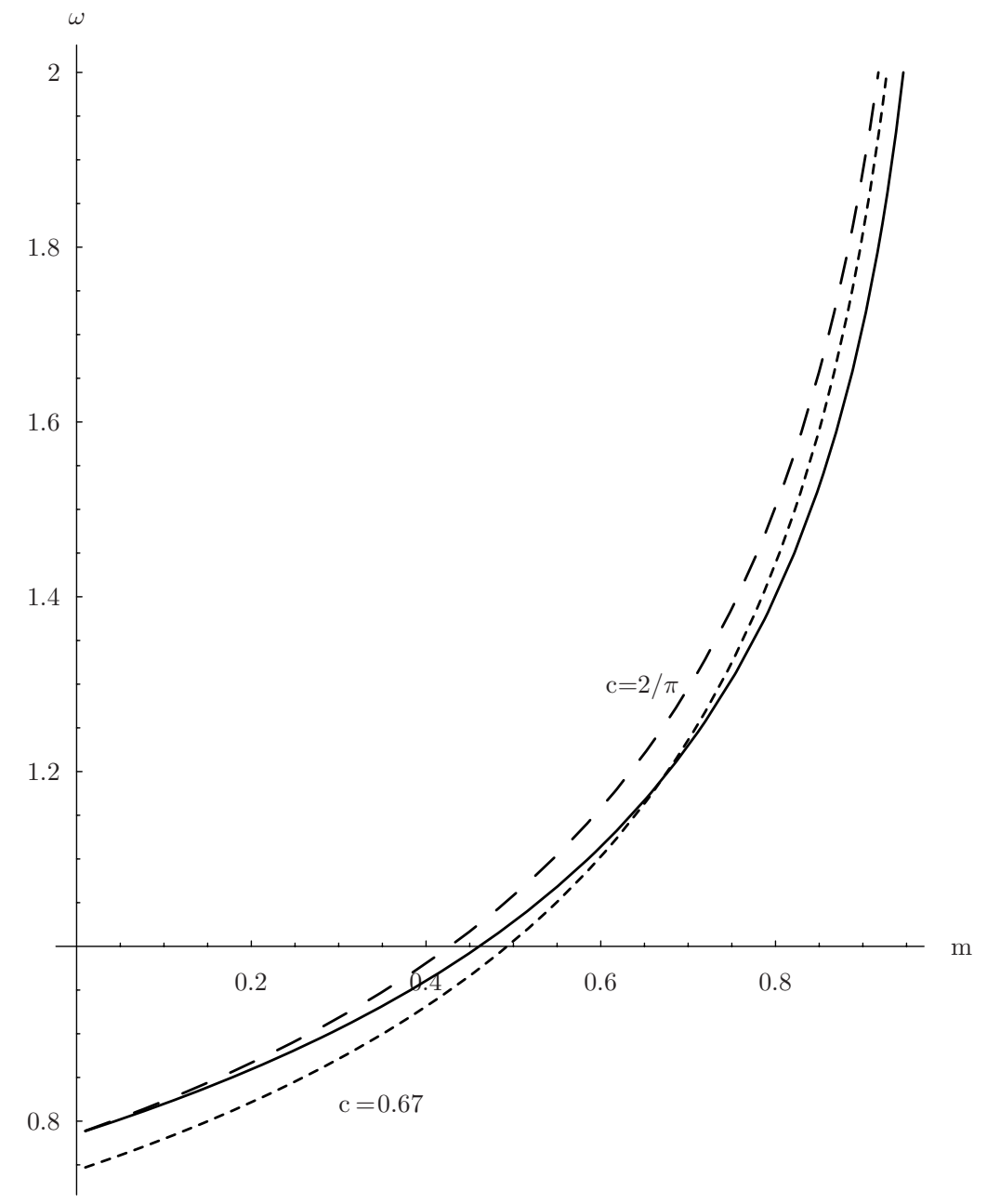

FiguRE 2. Level curves with $c=2 / \pi, c=0.67$, and their intersections with $\operatorname{sn}\left(\omega-K_{m}, m\right)=-1 / \sqrt{2}$.

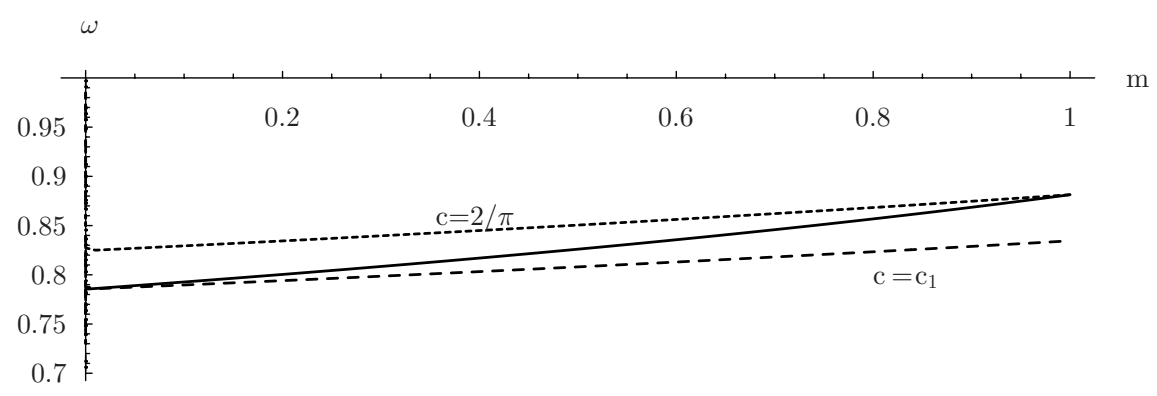

Figure 3. Level curves with $c=c_{1}, c=2 / \pi$, and their intersections with $\operatorname{sn}(\omega, m)=1 / \sqrt{2}$. 


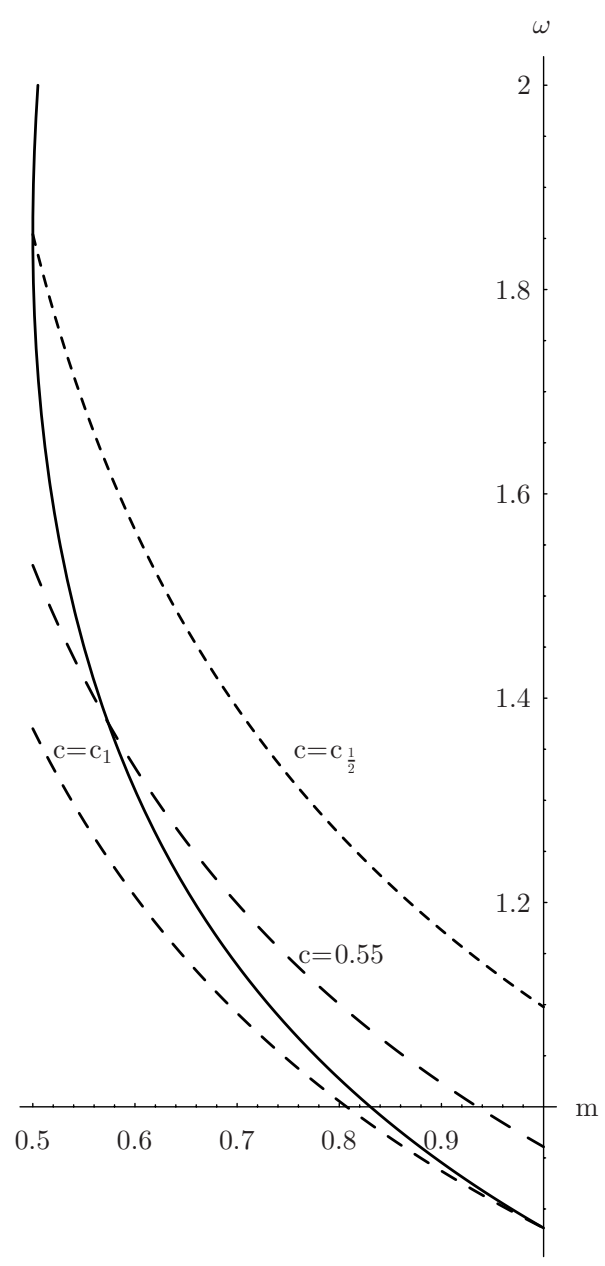

FIGURE 4. Level curves with $c=c_{\frac{1}{2}}, c=0.55, c=c_{1}$, and their intersections with $\sqrt{m} \operatorname{sn}(\omega, m)=1 / \sqrt{2}$.

where the constant of integration is zero since $\theta(0)=0$ is required. The value of $\mu_{1}$ is such that $\lambda \int_{0}^{1} \sin \theta(s) d s=\mu_{0}+\mu_{1}$, and therefore the equation rewrites as

$$
\theta(t)-\theta(0)-\lambda\left(\int_{0}^{t} \int_{0}^{s} \sin \theta(u) d u d s-(1+t) \int_{0}^{1} \sin \theta(s) d s\right)-\mu_{0}-\mu_{1}(1+t)=0,
$$

and hence the projected gradient vector field vanishes. The reasoning is almost identical when $c$ is such that one or the other of the two remaining systems (4.2), and (4.3), is involved.

It is time to return to the case of graphs with unconstrained length and present the main result. The global minimum corresponds to the case $\eta=0$ in Theorem 3.3 dealing with the critical points; see Figure 1 . With $m=1 / 2$, let

$$
\hat{L}=1 /\left(\frac{2 E_{m}}{K_{m}}-1\right) \approx 2.18844,
$$


and observe that the function $x(s)=\hat{L}\left(\frac{2 E\left(K_{m} s, m\right)}{K_{m}}-s\right)$ satisfies $x(0)=0, x(1)=1$. The derivative $x^{\prime}(s)=\hat{L}\left(2 \operatorname{dn}^{2}\left(K_{m} s, m\right)-1\right)$ is strictly positive in $[0,1)$. Denote the inverse of $x(s)$ by $s(x)$.

Theorem 4.3. There is a unique graph of minimal elastic energy given by

$$
\hat{y}(x)=\frac{\sqrt{2}}{\left(2 E_{\frac{1}{2}}-K_{\frac{1}{2}}\right)}\left(1-\operatorname{cn}\left(K_{\frac{1}{2}} s(x), \frac{1}{2}\right)\right)
$$

among graphs that can be parameterized as $(x(t), y(t))$ so that $(x(0), y(0))=(0,0)$, $x(1)=1$ and $\left(x^{\prime}(t), y^{\prime}(t)\right)=L(\cos \theta(t), \sin \theta(t))$, where $(\theta, L) \in W_{1}^{2}[0,1] \times \mathbb{R}^{+}$and $\theta(0)=0, \theta(1)=\pi / 2$.

Note that $\hat{y}(0)=0$ and $\hat{y}(1) \approx 1.66925$.

Proof. To prove this, consider each length $L>0$ and the corresponding $c=1 / L \in$ $(0,1)$. The idea of attaching a straight vertical piece at the right endpoint shows that the energy can only decrease or stay the same as $c$ decreases. It is shown in Theorem 4.2 above that the energy is constant on $\left(0, c_{\frac{1}{2}}\right]$. In this case the optimal curve, when $0<c<c_{\frac{1}{2}}$, is constructed by adding a straight segment at the right endpoint, which does not change the energy. It is now necessary to show that the energy is always greater than the constant value on $\left(0, c_{\frac{1}{2}}\right]$ if $c>c_{\frac{1}{2}}$. It suffices to restrict the attention to the interval $\left(c_{\frac{1}{2}}, c_{1}\right)$. On this interval solve the less restrictive problem without the constraint $|\theta(t)| \leqslant \pi / 2$. Recall that for each $c \in\left(c_{\frac{1}{2}}, c_{1}\right)$ there exists a curve of minimal elastic energy in this case, and the curve must satisfy

$$
\theta(t)-\lambda \int_{0}^{t} \int_{0}^{s} \sin \theta(u) d u d s+t \mu_{0}=0
$$

This equation forces the minimizing $\theta$ to be $C^{\infty}$ smooth. Moreover, when $c \in$ $\left(c_{\frac{1}{2}}, c_{1}\right)$, the minimizing curves satisfy $|\theta(t)| \leqslant \pi / 2$. Now suppose $c \in\left(c_{\frac{1}{2}}, c_{1}\right)$ and let $\theta_{c}$ be the minimizing solution in this case. Let $c^{\prime} \in\left(c_{\frac{1}{2}}, c_{1}\right)$ be such that $c^{\prime}<c$. Attach a straight line segment to the right endpoint of the curve associated with $\theta_{c}$, and choose the length of the segment so that the length of this new curve satisfies $1 / L=c^{\prime}$. Since the corresponding curve is not $C^{\infty}$ smooth, it cannot have minimal energy. Specifically, the straight line segment has all derivatives equal to zero, so it is important here that the only solution to (4.10) with all derivatives equal to zero at some point is the zero solution, which is not admissible. It follows that there is some other curve $\theta^{\prime}$ of strictly smaller energy that satisfies the boundary conditions, and therefore the energy is strictly decreasing as $c$ decreases. Hence the minimal energy curves are produced by $c \in\left(0, c_{\frac{1}{2}}\right]$. Only $c=c_{\frac{1}{2}}$ yields a curve that is also a graph.

The explicit formula follows by integrating $\left(x^{\prime}(t), y^{\prime}(t)\right)=\hat{L}(\cos \theta(t), \sin \theta(t))$, where $\hat{L}=1 / c_{\frac{1}{2}}$.

\section{REFERENCES}

[1] Adams, R. A., Fournier, J. J. F., Sobolev Spaces (2nd. ed.), Academic Press (2003), (review of 1975 original edition) MR0450957 (56:9247)

[2] Birkhoff, G., deBoor C. R., Piecewise polynomial interpolation and approximation, Internat. J. Math. Approximation of Functions (Proc. Sympos. General Motors Res. Lab., 1964), Elsevier, Amsterdam (1965), 164-190 MR0189219 (32:6646)

[3] Bowman, F., Introduction to elliptic functions with applications, Dover (1961) MR0132214 (24:A2060) 
[4] Deckelnick, K., Grunau, H.-C., Boundary value problems for the one-dimensional Willmore equation - Almost explicit solutions, Technical Report Nr. 2, Univ. Magdeburg (2005)

[5] Fuss, P. H., Correspondance Mathématique et Physique de quelques célèbres géomètres du XVIIIème siècle (1843), volume 2. Johnson Reprint Corporation (1968)

[6] Ioffe, A. D., Tihomirov, V. M., Theory of Extremal Problems, Studies in Mathematics and its Applications, Vol. 6, North-Holland (1979) MR0528295 (80d:49001b)

[7] Jerome, J. W., Smooth interpolating curves of prescribed length and minimum curvature Proc. Amer. Math. Soc. 51 (1975), 62-66 MR0380551 (52:1451)

[8] Langer, J., Singer, D. A., Curve-straightening in Riemannian manifolds, Ann. Global Anal. Geom. 5 (1987), 133-150 MR0944778 (89i:58025)

[9] Linnér, A., Unified representations of non-linear splines, J. Approx. Theory 84 (1996), 315350 MR:1377607 (97f:41012)

[10] _ Existence of free non-closed Euler-Bernoulli elastica, Nonlinear Anal. 21 (1993), 575-593 MR1245863 (94m:58047)

[11] _ Riemannian Structures and Free Curve-Straightening, Media-Enhanced Mathematics, http://www.math.niu.edu/ alinner/MEM/(August 24, 2005)

[12] Yosida, K., Functional Analysis (5th. ed.), Springer (1978) MR0500055 (58:17765)

Department of Mathematical Sciences, Northern Illinois University, DeKalb, IlliNOIS 60115

E-mail address: alinner@math.niu.edu

Department of Mathematics, Northwestern University, Evanston, Illinois 60208

E-mail address: jwj@math.northwestern.edu 